\title{
A Fast Algorithm for Analysis of Molecular Communication in Artificial Synapse
}

\author{
Bilgesu A. Bilgin, Member, IEEE and Ozgur B. Akan, Fellow, IEEE
}

\begin{abstract}
In this paper we analyse molecular communications (MC) in a proposed artificial synapse (AS), whose main difference from biological synapses (BSs) is that it is closed, i.e., transmitter molecules cannot diffuse out from AS. Such a setup has both advantages and disadvantages. Besides higher structural stability, being closed, AS never runs out of transmitters. Thus, MC in AS is disconnected from outer environment, which is very desirable for possible intra-body applications. On the other hand, clearance of transmitters from AS has to be achieved by transporter molecules on the presynaptic membrane of AS. Except from these differences, rest of AS content is taken to be similar to that of a glutamatergic BS. Furthermore, in place of commonly used Monte Carlo based random walk experiments, we derive a deterministic algorithm that attacks for expected values of desired parameters such as evolution of receptor states. To assess validity of our algorithm we compare its results with average results of an ensemble of Monte Carlo experiments, which shows near exact match. Moreover, our approach requires significantly less amount of computation compared to Monte Carlo approach, making it useful for parameter space exploration necessary for optimisation in design of possible MC devices, including but not limited to AS. Results of our algorithm are presented in case of single quantal release only, and they support that $\mathrm{MC}$ in closed AS with elevated uptake has similar properties to that in BS. In particular, similar to glutamatergic BSs, the quantal size and density of receptors are found to be main sources of synaptic plasticity. On the other hand, the proposed model of AS is found to have slower decaying transients of receptor states compared to BSs, especially desensitised ones, which is due prolonged clearance of transmitters from AS.
\end{abstract}

Index Terms-Neuro-spike communication, Synaptic Channel, Diffusion, Receptor Binding, Synaptic variability

\section{INTRODUCTION}

Nature, the greatest engineer of all, uses molecules to create the most complex integrated systems. Deciphering its language necessarily requires the understanding of principles of $\mathrm{MC}$ in such integrated systems [1]. As our understanding of these

The paper has been published in IEEE Transactions on NanoBioscience on 21 July 2017. Paper available at http://ieeexplore.ieee.org/abstract/document/7987782/.

DOI: 10.1109/TNB.2017.2730582

(c) 2017 IEEE. Personal use of this material is permitted. Permission from IEEE must be obtained for all other uses, in any current or future media, including reprinting/republishing this material for advertising or promotional purposes, creating new collective works, for resale or redistribution to servers or lists, or reuse of any copyrighted component of this work in other works.

The authors are with the Next-generation and Wireless Communications Laboratory (NWCL), Department of Electrical and Electronics Engineering, Koc University, Istanbul, 34450, Turkey (e-mail: \{bilgesubilgin, akan\}@ku.edu.tr).

O. B. Akan is also with Electrical Engineering Division, Department of Engineering, University of Cambridge, UK (e-mail: oba21@ cam.ac.uk).

This work was supported in part by the ERC project MINERVA (ERC2013-CoG \#616922), and the EU project CIRCLE (EU-H2020-FET-Open \#665564).

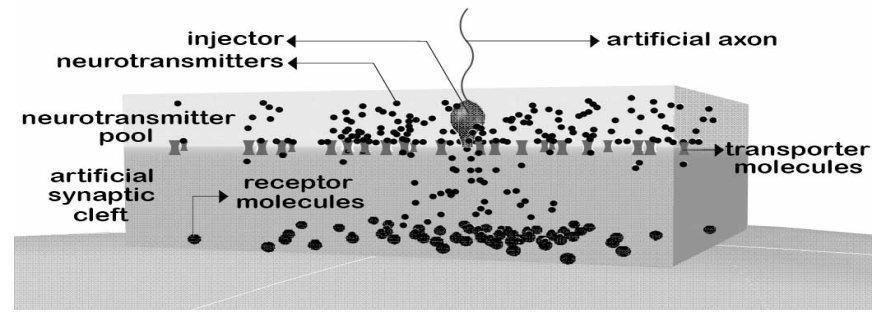

Fig. 1: AS is divided into two main parts, namely the neurotransmitter pool and the artificial synaptic cleft. Upon arriving impulse neurotransmitters are into synaptic cleft. As AS is bounded by walls, transmitters can't diffuse out and transporter molecules are only mechanism to clear AS from transmitters.

systems grows, so does our urge to manipulate and control them. Latter requires the ability to communicate with such systems, which implies that we need to design networks of nano-scale machines, i.e., nanomachines, capable of carrying out reliable MC with the constituents of these systems [2]. ICT based approaches developed for macro-scale networks are already being refined and utilized to tackle such design problems [3], [4]. If successful, these undertakings promise technological progress in fields such as targeted drug delivery [2], brain-machine interfaces [3] and disease diagnosis [5].

As the estimated number of neurons in an adult brain is comparable to that of the stars in our galaxy, one of the most complex of natural systems employing $\mathrm{MC}$ is the human nervous system [6]. In the nervous system, neurons communicate with each other by means of short pulses of electrical signals called action potentials. An action potential produced by a transmitter neuron travels along its axons, outwardly elongated projections of the neuron, which make junctions with a receiving neuron. These junctions are called synapses, and by structural considerations they divide into two main categories, electrical synapses and chemical synapses [7]. In electrical synapses, there is no gap in the junction, and the transmission of the electrical signal is achieved by means of ion flow through ion-channels sitting on the membranes of both the transmitting, or presynaptic, neuron and the receiving, or postsynaptic, neuron. On the other hand, chemical synapses have a small gap, called the synaptic cleft, at the junction where transmission of information occurs. In these synapses, the arriving action potential triggers a vesicle filled with a specific type of molecule, referred to as neurotransmitters, to release its contents into the synaptic cleft. Upon release, these transmitters diffuse inside the cleft, and attach to receptors on the postsynaptic membrane. This causes ion-channels to be opened on the postsynaptic membrane, resulting in flow 
of ions across the membrane. In both cases of synapses, the ion flow across the postsynaptic membrane causes the membrane potential to change. This change in the membrane potential either increases or decreases the probability that the postsynaptic neuron produces an action potential, depending on whether the synapse is an excitatory or inhibitory one, respectively. The synapses which cause more change in the postsynaptic neuron are said to be stronger.

From an information theoretical point of view, the chemical synapses are rather more complex, because they involve transduction of the transmitted signal from electrical to chemical, and then back to electrical form. This complexity is thought to be a main contributor of synaptic plasticity, the ability of synapses to change their strength, which is considered to be crucial for the processing capabilities of a neural system such as memory formation and learning [8], [9], [10]. For this reason, the focus of this paper will be on chemical synapses.

Our aim in this work is to introduce a primitive model of an $\mathrm{AS}$, and investigate the properties of MC inside it. In the literature, one can find many innovative designs of AS [11], [12], [13], [14]. All of these designs are significant in that they are all realized machines of very small size (but not nanoscale) capable of, to some extent, mimicking the behavior of a real biological synapse. However, on the down side, they are far from being compatible with biological neurons. A promising approach in terms of biocompatibility is presented in [15].

In this paper, we do not propose a realizable way of mimicking real synapses by means of an artificial one, but rather, we seek to consider the real synapse slightly modified, and try to understand the effects of this modification on the MC properties of the synaptic communication channel. Thus, the main difference of our AS from the biological synapses is that, the AS is bounded by impermeable walls, as depicted in Fig.1. Hence, the transmitter molecules are trapped inside a closed box, which makes the MC inside the AS independent of phenomena outside the AS. This independence is a desirable property for an AS. It not only maximizes storage capacity and permits sparse coding at individual synapses, but also reduces the risk of bio-incompatibility in possible intra-body applications. Moreover, as no transmitters get lost to the surrounding, the transmitter reserves of the AS are never depleted. Furthermore, the existence of enclosing walls provides structural stability by establishing contact with the artificial postsynaptic neuron. All of these properties simplify the possible physical realization of such an AS.

On the other hand, the fact that our AS is a closed system, brings about a crucial disadvantage compared to biological synapses. In biological synapses, except those in neuromuscular junctions, where acetylcholine is deactivated by enzymatic degradation [16], the clearance of the transmitters are due to rapid diffusion out of the cleft [17], [18]. Obviously, such a mechanism lacks for the proposed AS. Thus, in order to achieve clearance of transmitters from AS, we place a high density of transporter molecules on the presynaptic membrane of AS.

Except the differences mentioned, the molecular content of $\mathrm{AS}$ is taken to be the same as that of a glutamatergic synapse.
The postsynaptic membrane is assumed to contain $\alpha$-amino3-hydroxy-5methyl-isoxazolepropionic acid receptors (AMPARs) and $N$-methyl-D-aspartate receptors (NMDARs), which are known to be localized at many glutamatergic synapses [19], [20], [21], [22], [23]. Glutamate uptake in biological glutamatergic synapses is known to be preformed by at least five different transporter proteins [24]. We assume only one type of glutamate transporter (GluT) to occupy the presynaptic wall of AS.

In order to investigate the properties of the MC of our AS, instead of simulating via Monte Carlo based random walk experiments, we develop by means of mathematical analysis a deterministic code. This code directly calculates the expected values of desired variables, such as the glutamate concentration inside AS and the occupancy rates of receptor states. This approach has the advantage of determining the expected behavior of AS in a single run-time as opposed to the many experiments required in the Monte Carlo method. It also causes significantly less energy consumption and permits a faster and cheaper method to explore the parameter space.

The remainder of the paper is organized as follows. In Section II we first give the details of our physical model of the AS, where we introduce the nominal values used for the physical dimensions of the AS, as well as the quantities of the types of molecules used and their reaction kinematics with each other. All these parameters are first chosen as in [25] to enable the opportunity of comparison of results of our method with that of the Monte Carlo method used there. However, as [25] deals with biological glutamatergic synapses, we modify values of some parameters later to obtain a desired response from our AS. Next, in Section II-B we analytically derive the partial differential equation (PDE) together with its boundary conditions, which governs the MC inside the AS. After that, in Section II-B2, we devise a numerical scheme based on a finite difference method, which we show to be an approximation to the PDE under given boundary conditions. The analytical scheme developed is the basis of our code, with which we predict the MC in AS. Finally, in Section II-C we evaluate the performance by comparing it to the approach employed in [25], and confirm the validity of our code by comparing its results to the average of the results of a series of Monte Carlo experiments. Section III is devoted to the presentation and interpretation of the results of the algorithm developed in Section II. Our aim here mainly is to demonstrate that for a wide part of the parameter space, the AS shows behavior similar to a biological synapse, that is the lack of clearance of glutamates due to diffusion can be compensated by an elevated population of GluTs on the presynaptic membrane. Finally, in Section IV, we conclude by summarizing our findings and point out directions for future research.

\section{THE MODEL}

\section{A. The Artificial Synapse}

As was mentioned in Section I, our AS is bounded by the vertical reflective walls, see Fig.1, which are assumed to be devoid of any receptor or transporter molecules. The nominal values used for all parameters of AS are presented in Table 
I. All nominal values, except that of the GluT density, fall into the range of typical values for a biological neuron, and the nominal value of GluT density used is encountered on the membranes of hippocampal astroglia, although not on the presynaptic membrane of glutamatergic synapses [25]. The AS is assumed to have the shape of a rectangular box with a $0.5 \times 0.5 \mu \mathrm{m}^{2}$ quadratic base, and a height of $20 \mathrm{~nm}$. We are not interested in the dimensions and configuration of the part of the AS illustrated in Fig.1 as the transmitter pool. All we assume is that, upon the arrival of an electric pulse via the artificial axon, the injector injects a prescribed amount of transmitters into the AS.

TABLE I: Nominal values of AS parameters.

\begin{tabular}{ll}
\hline Parameter & Value \\
\hline Base area of AS & $0.5 \times 0.5 \mu \mathrm{m}^{2}$ \\
Height of AS cleft & $20 \mathrm{~nm}$ \\
Diameter of the PSD & $350 \mathrm{~nm}$ \\
Number of AMPARs & 80 \\
Number of NMDARs & 20 \\
GluT density & 10000 molecules $/ \mu \mathrm{m}^{2}$ \\
Quantal size & 3000 \\
Diffusion rate constant, D & $0.2 \mu \mathrm{m}^{2} / \mathrm{ms}$ \\
\hline
\end{tabular}

The molecular content of the AS is assumed to be similar to that of a biological glutamatergic synapse. We uniformly distribute AMPARs and NMDARs on the postsynaptic density (PSD), which covers a disk of diameter $350 \mathrm{~nm}$ on the postsynaptic membrane, with nominal values of 80 AMPARs and 20 NMDARs, unless specified otherwise. For the reaction schemes of AMPAR and NMDAR with glutamate we use the kinematic models illustrated in Fig.2(a) and 2(b) as in [26] and [27], respectively. According to these models, AMPAR and NMDAR require two glutamate molecules to reach the open state. The GluTs are uniformly spread over all of the presynaptic membrane with a nominal density of 10000 molecules per $\mu \mathrm{m}^{2}$. The GluTs are assumed to obey a simple three-state reaction scheme with a slow turnover rate [28], also used in [29] and [30]. The rate constants for all these kinematic models are provided in Table II. The nominal quantal size is taken to be 3000 molecules per transmitter release event unless specified otherwise. The time-course of glutamate release is very rapid, [31], and therefore, we assume that the transmitter release is instantaneous, and also point-wise.

The glutamate diffusion rate constant, $D$, inside the AS is taken as $0,2 \mu \mathrm{m}^{2} \mathrm{~ms}^{-1}$, [32], approximately one-third of that of aqueous glutamine due to molecular overcrowding, [33], [34], [35].

\section{B. Mathematical Analysis and Numerical Scheme}

1) Mathematical Analysis: The diffusion of the glutamate molecules inside the synaptic cleft is modeled by the celebrated diffusion equation

$$
\partial_{t} u(\vec{x}, t)=D \Delta u(\vec{x}, t), \quad \vec{x} \in \Omega, t>0,
$$

where $D$ is the diffusion rate constant, $\Omega$ corresponds to the inside of the synaptic cleft, $\partial_{t}$ stands for the partial derivative with respect to time, and $\Delta=\sum_{i=1}^{3} \partial_{x_{i}}^{2}$ with the notation $\vec{x}=$

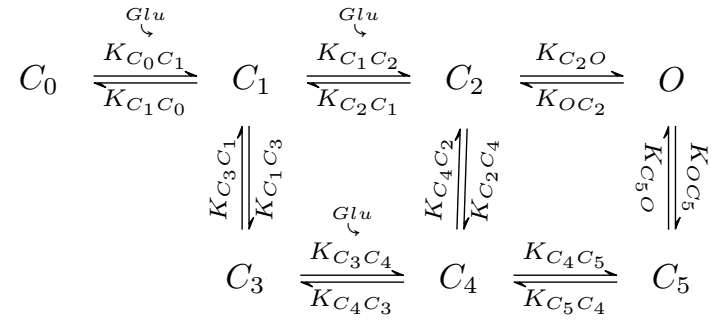

(a) AMPAR reaction scheme.

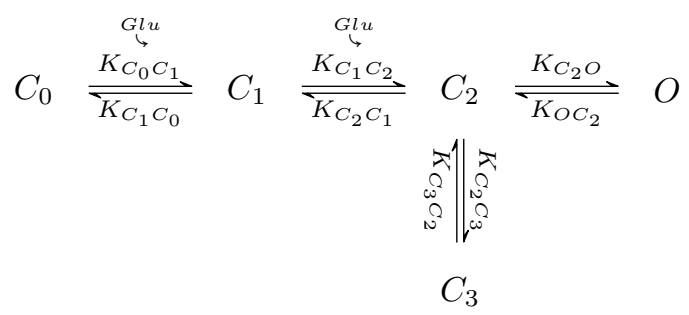

(b) NMDAR reaction scheme

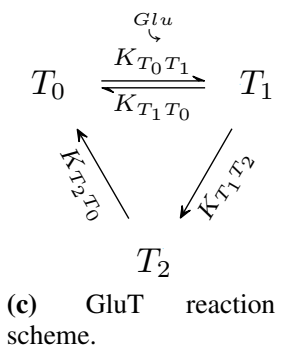

Fig. 2: Reaction schemes for the assumed kinetic models.

$\left(x_{1}, x_{2}, x_{3}\right) \cdot u(\vec{x}, t)$ is the expected concentration of glutamate molecules at the point $\vec{x}$ at time $t$, and therefore, the expected number of glutamates found in a volume $V \subset \Omega$ at time t, $N_{V}(t)$, is given by

$$
N_{V}(t)=\int_{V} u(\vec{x}, t) d v, \quad d v=\prod_{i=1}^{3} d x_{i}
$$

We use the notation $N(t):=N_{\Omega}(t)$ for the expected number of all free glutamate molecules left inside the artificial synaptic cleft.

We need to supplement (1) with boundary conditions, which should be determined by the characteristics and distribution of the receptors and transporters on the boundary that interact with the glutamate molecules. In order to derive these conditions, we first notice that, the rate of change in the number of free glutamate molecules inside $\Omega$ is related to the gradient of the concentration at the boundary. This follows upon integration of equation (1) over $\Omega$. Thanks to the Stoke's theorem one obtains

$$
\frac{d}{d t} N(t)=D \int_{\partial \Omega} \nabla u(\sigma, t) \cdot \vec{n}(\sigma) d \sigma
$$

where $\partial \Omega$ is the boundary of $\Omega$, and $\vec{n}(\sigma)$ is the outward unit normal vector at the boundary point $\sigma$. Thus, the number of glutamate molecules we lose to a boundary patch $\Gamma \subset \partial \Omega$ over 
TABLE II: Rate constants for the kinetic models.

\begin{tabular}{|c|c|c|c|}
\hline Parameter & Symbol & Value & Ref. \\
\hline $\begin{array}{l}\text { AMPA Receptors } \\
\text { First association rate constant } \\
\text { First dissociation rate constant } \\
\text { Second association rate constant } \\
\text { Second dissociation rate constant } \\
\text { Channel opening rate constant } \\
\text { Channel closing rate constant } \\
C_{1} \text { to } C_{3} \text { desensitization rate constant } \\
C_{3} \text { to } C_{1} \text { resensitization rate constant } \\
C_{3} \text { to } C_{4} \text { association rate constant } \\
C_{4} \text { to } C_{3} \text { dissociation rate constant } \\
C_{2} \text { to } C_{4} \text { desensitization rate constant } \\
C_{4} \text { to } C_{2} \text { resensitization rate constant } \\
C_{4} \text { to } C_{5} \text { isomerization rate constant } \\
C_{5} \text { to } C_{4} \text { isomerization rate constant } \\
\text { Open to } C_{5} \text { desensitization rate constant } \\
C_{5} \text { to Open resensitization rate constant }\end{array}$ & $\begin{array}{l}K_{C_{0} C_{1}} \\
K_{C_{1} C_{0}} \\
K_{C_{1} C_{2}} \\
K_{C_{2} C_{1}} \\
K_{C_{2} O} \\
K_{O C_{2}} \\
K_{C_{1} C_{3}} \\
K_{C_{3} C_{1}} \\
K_{C_{3} C_{4}} \\
K_{C_{4} C_{3}} \\
K_{C_{2} C_{4}} \\
K_{C_{4} C_{2}} \\
K_{C_{4} C_{5}} \\
K_{C_{5} C_{4}} \\
K_{O C_{5}} \\
K_{C_{5} O}\end{array}$ & $\begin{array}{l}4.59 \times 10^{6} \mathrm{M}^{-1} \mathrm{~s}^{-1} \\
4.26 \times 10^{3} \mathrm{~s}^{-1} \\
2.84 \times 10^{6} \mathrm{M}^{-1} \mathrm{~s}^{-1} \\
3.26 \times 10^{3} \mathrm{~s}^{-1} \\
4.24 \times 10^{3} \mathrm{~s}^{-1} \\
900 \mathrm{~s}^{-1} \\
2.89 \times 10^{3} \mathrm{~s}^{-1} \\
39.2 \mathrm{~s}^{-1} \\
1.27 \times 10^{6} \mathrm{M}^{-1} \mathrm{~s}^{-1} \\
45.7 \mathrm{~s}^{-1} \\
172 \mathrm{~s}^{-1} \\
0.727 \mathrm{~s}^{-1} \\
16.8 \mathrm{~s}^{-1} \\
190.4 \mathrm{~s}^{-1} \\
17.7 \mathrm{~s}^{-1} \\
4.0 \mathrm{~s}^{-1}\end{array}$ & [26] \\
\hline $\begin{array}{l}\text { NMDA Receptors } \\
\text { First association rate constant } \\
\text { First dissociation rate constant } \\
\text { Second association rate constant } \\
\text { Second dissociation rate constant } \\
\text { Channel opening rate constant } \\
\text { Channel closing rate constant } \\
C_{2} \text { to } C_{3} \text { desensitization rate constant } \\
C_{3} \text { to } C_{2} \text { resensitization rate constant }\end{array}$ & $\begin{array}{l}K_{C_{0} C_{1}} \\
K_{C_{1} C_{0}} \\
K_{C_{1} C_{2}} \\
K_{C_{2} C_{1}} \\
K_{C_{2} O} \\
K_{O C_{2}} \\
K_{C_{2} C_{3}} \\
K_{C_{3} C_{2}}\end{array}$ & $\begin{array}{l}2 \times 5.0 \times 10^{6} \mathrm{M}^{-1} \mathrm{~s}^{-1} \\
4.7 \mathrm{~s}^{-1} \\
5.0 \times 10^{6} \mathrm{M}^{-1} \mathrm{~s}^{-1} \\
2 \times 4.7 \mathrm{~s}^{-1} \\
46.5 \mathrm{~s}^{-1} \\
91.6 \mathrm{~s}^{-1} \\
8.4 \mathrm{~s}^{-1} \\
1.8 \mathrm{~s}^{-1}\end{array}$ & [27] \\
\hline $\begin{array}{l}\text { Glutamate Transporters } \\
\text { Association rate constant } \\
\text { Dissociation rate constant } \\
\text { Transition state rate constant } \\
\text { Transport rate constant }\end{array}$ & $\begin{array}{l}K_{T_{0} T_{1}} \\
K_{T_{1} T_{0}} \\
K_{T_{1} T_{2}} \\
K_{T_{2} T_{0}}\end{array}$ & $\begin{array}{l}18.0 \times 10^{6} \mathrm{M}^{-1} \mathrm{~s}^{-1} \\
180 \mathrm{~s}^{-1} \\
180 \mathrm{~s}^{-1} \\
25.7 \mathrm{~s}^{-1}\end{array}$ & [30] \\
\hline
\end{tabular}

the time interval $(t, t+\Delta t)$ is given by

$$
n_{\Gamma}(t, t+\Delta t)=-D \int_{t}^{t+\Delta t} \int_{\Gamma} \nabla u(\sigma, \tau) \cdot \vec{n}(\sigma) d \sigma d \tau
$$

Now, we turn our attention to the boundary itself. As explained before, the boundaries of our artificial synapse contain AMPA and NMDA receptors, as well as glutamate transporters. The kinetic models used for the reactions of these molecules with glutamate are presented in Fig.2 with corresponding rate constants supplemented in Table II.

Let us now derive the amount of free glutamate molecules that are captured by a given receptor. Let $R$ be a receptor with a fixed kinematic model with states $S_{i}, i=1, \cdots, r$. Observe that, for a given receptor the loss of free glutamates to that receptor occurs only during transitions between certain states, i.e., during transitions $C_{0} \rightarrow C_{1}, C_{1} \rightarrow C_{2}$ and $C_{3} \rightarrow C_{4}$ for AMPAR, $C_{0} \rightarrow C_{1}$ and $C_{1} \rightarrow C_{2}$ for NMDAR, and during $T_{0} \rightarrow T_{1}$ for GluT. All such state transitions are referred to as adsorbing state transitions, and their inverses are called releasing state transitions, since either a glutamate is adsorbed or released during these transitions. The common theme in all adsorbing state transitions is that the transition rate linearly depends on the glutamate concentration around the receptor with the linearity coefficient equal to the rate constant given in Table II. For each $i, j=1, \cdots, r$, let us denote by $\kappa_{i j}$ the transition rate constant from state $S_{i}$ to $S_{j}$ in units $s^{-1}$, that is for adsorbing transitions the correct transition rate constant is found by multiplying the concentration of glutamates near the receptor by the rate constant given in Table II, which is in units $M^{-1} s^{-1}$. We use the convention that $\kappa_{i j}=0$ if there is no transition from the state $S_{i}$ to $S_{j}$. In particular, we have $\kappa_{i i}=0$ for each $i=1, \cdots, r$, signified by the fact that there is no rate constant in Table II in the form $K_{i i}$. Then, $P_{R, i}(t)$, the probability that the receptor $R$ is in state $S_{i}$ at time $t$, satisfies the following

$$
\frac{d}{d t} P_{R, i}(t)=-\sum_{j=1}^{r} \kappa_{i j} P_{R, i}(t)+\sum_{j=1}^{r} \kappa_{j i} P_{R, j}(t), \quad i=1, \cdots, r .
$$

With the notation $P_{R}(t):=\left(P_{R, 1}(t), \cdots, P_{R, r}(t)\right)^{T}$ and the convention on $\kappa_{i j}$ 's, these equations can be written in the more compact form

$$
\frac{d}{d t} P_{R}(t)=A_{R}(t) P_{R}(t)
$$

where $A_{R}(t)$ is a $r \times r$-matrix with coefficients

$$
\left(A_{R}\right)_{i j}=\left\{\begin{array}{cc}
-\sum_{k=1}^{r} \kappa_{i k} & , \text { if } i=j, \\
\kappa_{j i} & , \text { if } i \neq j,
\end{array}\right.
$$

and is called the state transition matrix of the receptor $R$. As an example, the state transition matrix of a GluT at position $\sigma \in \partial \Omega$ at time $t$ is

$$
A_{G l u T}=\left[\begin{array}{ccc}
-u(\sigma, t) K_{T_{0} T_{1}} & K_{T_{1} T_{0}} & K_{T_{2} T_{0}} \\
u(\sigma, t) K_{T_{0} T_{1}} & -K_{T_{1} T_{0}}-K_{T_{1} T_{2}} & 0 \\
0 & K_{T_{1} T_{2}} & -K_{T_{2} T_{0}}
\end{array}\right] .
$$

Observe that in all columns of $A_{G l u T}$ the sum of the entries is zero. This is generally true for all state transition matrices, which easily follows by adding the equations in (3) over $i$, and corresponds to the fact that the sum of the entries of the probability vector $P_{R}(t)$ remains constant in time. Thus, starting at $t_{0}$ with an initial state distribution such that

$$
\sum_{i=1}^{r} P_{R, i}(0)=1
$$

the solution to (4) will satisfy

$$
\sum_{i=1}^{r} P_{R, i}(t)=1, \quad \forall t \geq 0 .
$$

In view of (4), the evolution of the occupancy probabilities of the states of a given receptors $R$ satisfies

$$
P_{R}(t+\Delta t)=\exp \left(\int_{t}^{t+\Delta t} A_{R}(\tau) d \tau\right) P_{R}(t), \quad t, \Delta t \geq 0 .
$$

The expected number of free glutamates a given receptor $R$ captures during the time interval $(t, t+\Delta t), n_{R}(t, t+\Delta t)$, can be found by keeping track of the adsorbing and releasing state transitions. We have

$n_{R}(t, t+\Delta t)=\left(\sum_{a d s .}-\sum_{r e l .}\right)\left(\exp \left(\int_{t}^{t+\Delta t} A_{R}(\tau) d \tau\right)\right)_{j i} P_{R, i}(t)$,

where the sums are over adsorbing and releasing state transitions. If we denote by $f_{R}$ the distribution of the receptor of type $R$ on the boundary, then the expected number of glutamates captured by a given patch on the boundary, $\Gamma \subset \partial \Omega$, during the time interval $(t, t+\Delta t)$ can be written as

$$
n_{\Gamma}(t, t+\Delta t)=\sum_{R} \int_{\Gamma} n_{R}(t, t+\Delta t) f_{R}(\sigma) d \sigma .
$$


where the sum is over the types AMPAR, NMDAR and GluT. Comparing (2) and (8), in view of (7), we deduce the following relation on the boundary

$$
\begin{gathered}
-D \int_{t}^{t+\Delta t} \nabla u(\sigma, \tau) \cdot \vec{n}(\sigma) d \tau= \\
\sum_{R}\left(\sum_{\text {ads }}-\sum_{r e l}\right)\left(\exp \left(\int_{t}^{t+\Delta t} A_{R}(\sigma, \tau) d \tau\right)\right)_{j i} P_{R, i}(\sigma, t) f_{R}(\sigma)
\end{gathered}
$$

valid for all $\sigma \in \partial \Omega$ and $t, \Delta t \geq 0$. Finally, differentiating (9) with respect to $\Delta t$ and evaluating at $\Delta t=0$, we deduce the following boundary condition

$$
\begin{aligned}
& -D \nabla u(\sigma, t) \cdot \vec{n}(\sigma)= \\
& \sum_{R}\left(\sum_{\text {ads }}-\sum_{r e l}\right)\left(A_{R}(\sigma, t)\right)_{j i} P_{R, i}(\sigma, t) f_{R}(\sigma) .
\end{aligned}
$$

To write this boundary condition in a more explicit form observe that, all state transitions, and in particular adsorbing and releasing state transitions, happen between different states, which implies that in all terms that appear in (10) we have $i \neq j$, and consequently from (5) we see that

$$
\left(A_{R}(\sigma, t)\right)_{j i}=\kappa_{i j}(\sigma, t) .
$$

Moreover, for the releasing state transitions the transition rate constant $\kappa_{i, j}$ is equal to the corresponding rate constant provided in Table II, which we denote as $K_{i j}$, and is therefore constant. However, for the adsorbing state transitions we have

$$
\kappa_{i j}(\sigma, t)=u(\sigma, t) K_{i j}, \quad \sigma \in \partial \Omega, t \geq 0 .
$$

Thus, more explicitly the boundary condition (10) may be rewritten as

$$
-D \nabla u(\sigma, t) \cdot \vec{n}(\sigma)=C_{a d s}(\sigma, t) u(\sigma, t)-C_{r e l}(\sigma, t),
$$

where

$$
C_{a d s, r e l}(\sigma, t)=\sum_{R} \sum_{a d s, r e l} K_{i j} P_{R, i}(\sigma, t) f_{R}(\sigma) .
$$

Note that, as $P_{R, i}$ depend nonlinearly on the concentration at the boundary, the boundary condition given above is highly nonlinear. Observe also that, as all the receptors lie on the two horizontal boundaries, see Fig.1. Therefore, the receptor distributions $f_{R}$ vanish at the vertical boundaries, and the condition (11) for these boundary components reduces to the Neumann boundary condition

$$
\nabla u(\sigma, t) \cdot \vec{n}(\sigma)=0 .
$$

2) Numerical Scheme: We divide our rectangular artificial synapse into smaller rectangles of sidelengths $\Delta x_{i}, i=1,2,3$. We take the midpoints of these small rectangles as the points of our grid, on which we employ the numerical scheme described below.

It is well-known that, (1) can be obtained as the limit of a discrete Brownian random walk on a 3-dimensional regular grid, which also corresponds to the discretization of (1) on the grid. Indeed, consider the discrete random walk of a single particle, whose progression of the occupancy probabilities of the grid points, $u[\vec{x}, t]$ ( $\vec{x}$ is now an integer valued vector), is given by the iterative formula

$$
\begin{aligned}
& u[\vec{x}, t+\Delta t]=\left(1-\sum_{i=1}^{3} \frac{2 D \Delta t}{\Delta x_{i}^{2}}\right) u[\vec{x}, t] \\
& +D \Delta t \sum_{i=1}^{3} \frac{u\left[\vec{x}+\vec{e}_{i}, t\right]+u\left[\vec{x}-\vec{e}_{i}, t\right]}{\Delta x_{i}^{2}}
\end{aligned}
$$

where $\vec{e}_{i}$ corresponds to the vector with the $i^{t h}$ entry equal to one and rest to zero, $i=1,2,3$. In order to assure the positivity of occupancy probabilities in (13) one also needs to choose the time and space increments as to satisfy

$$
\sum_{i=1}^{3} \frac{2 D \Delta t}{\Delta x_{i}^{2}}<1
$$

(13) can equivalently be rewritten as

$$
\begin{aligned}
& \frac{u[\vec{x}, t+\Delta t]-u[\vec{x}, t]}{\Delta t}= \\
& D \sum_{i=1}^{3} \frac{u\left[\vec{x}+\vec{e}_{i}, t\right]-2 u[\vec{x}, t]+u\left[\vec{x}-\vec{e}_{i}, t\right]}{\Delta x_{i}^{2}} .
\end{aligned}
$$

(15) together with the assumption that the paths of the particles are independent of each other, i.e., there are no collisions, gives us (1) in the limit as $\Delta x, \Delta t \rightarrow 0$.

The iterative formula (13) is valid only for the interior points of the grid. To derive the iteration formula for the points on the boundary, we first need to keep track of the receptor states, which is done by discretizing (6)

$$
P_{R}(t+\Delta t)=\exp \left(A_{R}(t) \Delta t\right) P_{R}(t), \quad t, \Delta t \geq 0 .
$$

Consequently, $n_{\delta}(\sigma, t, \Delta t)$, the density function on the boundary for the number of free glutamates captured by the receptors during the time interval $(t, \Delta t)$ can be approximated as

$$
\begin{aligned}
& n_{\delta}(\sigma, t, \Delta t)= \\
& \sum_{R}\left(\sum_{a d s}-\sum_{r e l}\right)\left(\exp \left(A_{R}(\sigma, t) \Delta t\right)\right)_{j i} P_{R, i}(\sigma, t) f_{R}(\sigma) .
\end{aligned}
$$

In order to write the iteration formula for the boundary points of our grid, let the integer valued vector $\vec{x}=\left(x_{1}, x_{2}, x_{3}\right)$ denote the position of a boundary grid point, and let $I \subset$ $\{1,2,3\}$ denote the directions in which $\vec{x}$ sits at the boundary. Furthermore, for the sake of ease of presentation suppose that $\left\{x_{i}\right\}_{i \in I}$ are maximal, i.e., our grid does not contain points with coordinates $x_{i}+1$. With this notation, the iterative formula for the boundary points of our grid reads

$$
\begin{aligned}
& u[\vec{x}, t+\Delta t]=\left(1-\sum_{i=1}^{3} \frac{2 D \Delta t}{\Delta x_{i}^{2}}\right) u[\vec{x}, t] \\
& +D \Delta t \sum_{i \notin I} \frac{u\left[\vec{x}+\vec{e}_{i}, t\right]+u\left[\vec{x}-\vec{e}_{i}, t\right]}{\Delta x_{i}^{2}} \\
& +D \Delta t \sum_{i \in I} \frac{u[\vec{x}, t]+u\left[\vec{x}-\vec{e}_{i}, t\right]}{\Delta x_{i}^{2}}-\sum_{i \in I} \frac{n_{\delta}(\sigma, t, \Delta t)}{\Delta x_{i}} .
\end{aligned}
$$


Indeed, (18) can be rewritten as

$$
\begin{aligned}
& \sqrt{\Delta t} \frac{u[\vec{x}, t+\Delta t]-u[\vec{x}, t]}{\Delta t}= \\
& D \sqrt{\Delta t} \sum_{i \notin I} \frac{u\left[\vec{x}+\vec{e}_{i}, t\right]-2 u[\vec{x}, t]+u\left[\vec{x}-\vec{e}_{i}, t\right]}{\Delta x_{i}^{2}} \\
& +\sum_{i \in I} \frac{D \sqrt{\Delta t}}{\Delta x_{i}}\left(-\frac{u[\vec{x}, t]-u\left[\vec{x}-\vec{e}_{i}, t\right]}{\Delta x_{i}}-\frac{n_{\delta}(\sigma, t, \Delta t)}{D \Delta t}\right) .
\end{aligned}
$$

Remember that, in (17) inside the sum the indices obey $i \neq j$, which implies

$$
n_{\delta}(\sigma, t, 0)=0
$$

As a consequence we have

$$
\lim _{\Delta t \searrow 0} \frac{n_{\delta}(\sigma, t, \Delta t)}{\Delta t}=\left.\frac{d}{d \Delta t} n_{\delta}(\sigma, t, \Delta t)\right|_{\Delta t=0},
$$

which is equal to the right hand side of the boundary condition (11) by the same calculations done there. Thus, taking limit above as $\Delta t, \Delta x_{i} \rightarrow 0$ with $\sqrt{\Delta t} / \Delta x_{i}$ kept constant, $i=1,2,3$, we find the boundary condition (11).

\section{Performance and Validation}

When implementing the numerical scheme described in the previous subsection, one has a restriction on the magnitudes of the spatial and temporal increments one can choose, namely the requirement that the inequality (14) must be satisfied. According to (14), for given values of spatial increments and the diffusion rate constant, one has an upper bound on the magnitude of the time increment as

$$
\Delta t<\frac{1}{2 D}\left(\sum_{i=1}^{3} \frac{1}{\Delta x_{i}^{2}}\right)^{-1} .
$$

Considering the value of $D$ and the dimensions of the AS given in Table I, in order to satisfy this restriction we choose our time-step as $0.1 \mu \mathrm{s}$. With this time-step calculating $10 \mathrm{~ms}$ of real-time required $\sim 250$ seconds for a $10 \times 10 \times 2$ grid, and $\sim 1000$ seconds on a $20 \times 20 \times 2$ grid on a single $2.1 \mathrm{GHz}$ PC workstation. The code was run on MATLAB.

For verification and performance comparison we have also run MCell, the Monte Carlo based molecular simulation program used by [25]. The results of the analytic approach and the average results of MCell experiments are overlaid in Fig. 8 for $10 \mathrm{~ms}$. of real-time. The comparison shows similar trends of receptor state evolution for both AMPA and NMDA receptors, although they are not an exact match. With a timestep of $0.1 \mu \mathrm{s}$, the simulation of $10 \mathrm{~ms}$ of real time took $\sim 1000$ seconds on the same workstation. Considering the fact that, the Monte Carlo approach requires a multitude of experiments to accurately obtain average results, we deduce that our approach is much faster and energy efficient. Another important point favoring our approach is the fact that our codes performance scales with the grid-size, where as any Monte Carlo experimental approach will scale with the number of molecules. Thus, in scenarios with high number of molecules or high density our approach becomes even more advantageous. and on a lower level programming language it should take significantly less time. Moreover, our code is highly parallelizable, as high as the number of spatial grid points, which ranged from 200 to 1600 in our calculations. We have pursued this direction as well, and parallellized our code. The parallellization is effective in decreasing the execution time for low number of cores, but ceases to be so for higher numbers, see Fig.3. The parallelization is done in spatial dimensions with each core specialized on a specific block. In order to carry out the necessary calculations for diffusion there is a need of information transfer amongst cores at the end of each timestep. This brought about an extra communication overhead, which diminished the effects of parallelization for high number of cores, rendering the pursuit for higher parallellization useless. To further understand the discrepancies between the

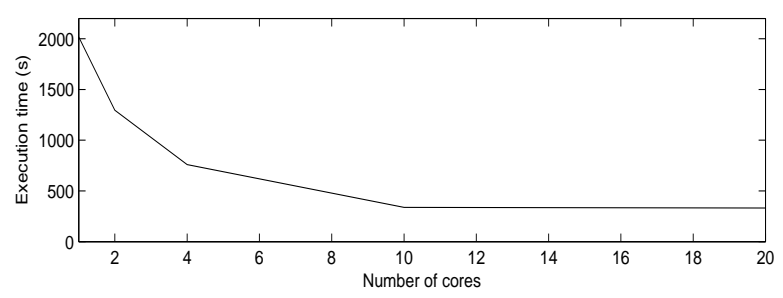

Fig. 3: The graph of the execution time of the code for a specific setup against varying number of cores used in parallel processing.

results of the analytic approach and MCell (Fig.8) and to provide further assessment to correctness of our simulation approach, we have written a separate code based on Monte Carlo approach, which simulates with the help of random number generators a simple random walk on a regular mesh. The reaction probabilities for this code were obtained again from (4) of our mathematical analysis. The comparison of the results are provided only for $10 \mathrm{~ms}$. of real time, mainly due to the bad performance of the Monte Carlo approach ( 1600 seconds for $1 \mathrm{~ms}$. real time with 0.1 microsec. time-step) combined with insufficiencies in the amount of available time and computational resources. For the evolution of AMPAR and NMDAR state occupancies, see Fig.7, shows a near perfect match between the analytic approach and the devised Monte Carlo approach (average of 100 experiments). This shows that the analytic approach accurately captures expected behavior of Monte Carlo experiments, and that the discrepancies observed in Fig.8 are mainly due to the different approaches of the Monte Carlo experiments. In our approach, diffusion process was carried out on a regular orthogonal grid, whereas in MCell there are no grids, and the random jump of the diffusing molecules are achieved by choosing a random direction and a jump distance. Moreover, the reactions in the analytic and Monte Carlo approach devised here always depend on the transmitter concentration inside the boundary grid where the reaction occurs. In contrast to this, in MCell reactions occur if transmitters hit the boundary inside a given radius around the receptor.

\section{RESULTS}

\section{A. Variations in GluT Density}

In synaptic MC it is essential that, soon after the quantal release the transmitters should be cleared out of the synaptic 

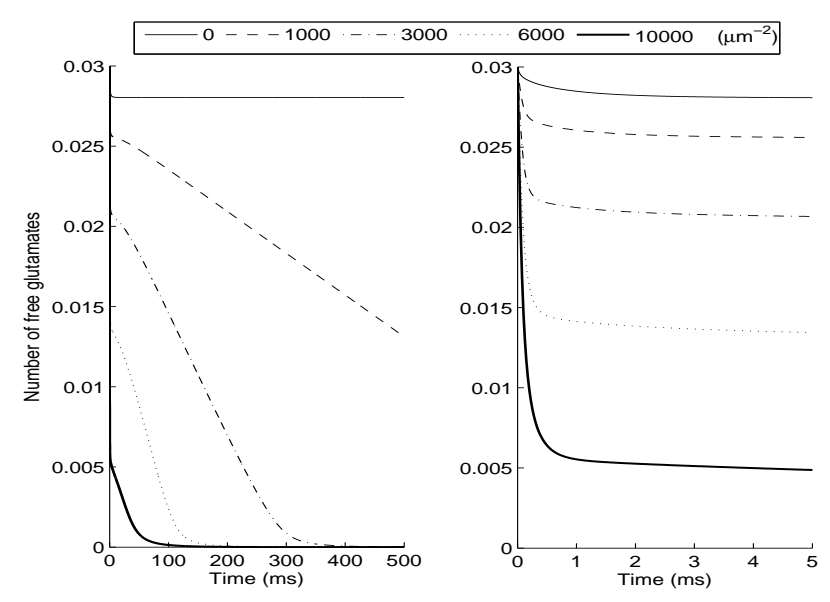

Fig. 4: Timecourse of free glutamate inside AS for different values of GluT density. The graph on the right is the zoomed in version of the one on left. The initial rapid capture of glutamates by GluTs slows down due to slow turnover rate of GluT.

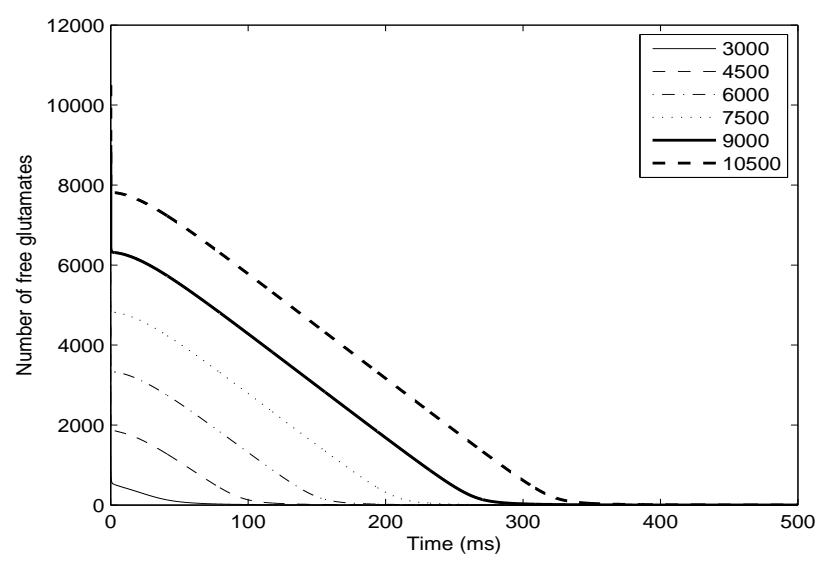

Fig. 5: Timecourse of free glutamate inside the cleft of AS for different values of quantal sizes. The clearance time of glutamates linearly depends on the quantal size for large quantal sizes.

cleft. Indeed, as was verified by the simulations of [25], the clearance time for the biological synapse is around $1 \mathrm{~ms}$. Our calculations for the AS reveal that, as in Fig.4, GluTs do, in fact, clear the AS of glutamates in $<1 \mathrm{~ms}$. However, due to the slow turnover rate of GluT, [28], the amount of this initial clearance is restricted to the number of GluTs on the presynaptic membrane of AS. Indeed, for example for the GluT density of 10000 molecules per $\mu \mathrm{m}^{2}$ our AS contains 2500 GluTs, which is exactly the amount of initial rapid decrease observed in Fig.4. After all GluTs capture a glutamate, it takes a long time for them to recapture another glutamate because of their slow turnover rate, and therefore the clearance slows down. Fig.5 shows that the clearance time linearly increases with quantal size, which seemingly makes things even worse. In view of this, it is reasonable to argue that glutamate clearance time from AS can be taken down to the level of a biological synapse by increasing the number of GluTs inside AS, which can be achieved either by increasing the GluT density or by increasing the base area of the AS.
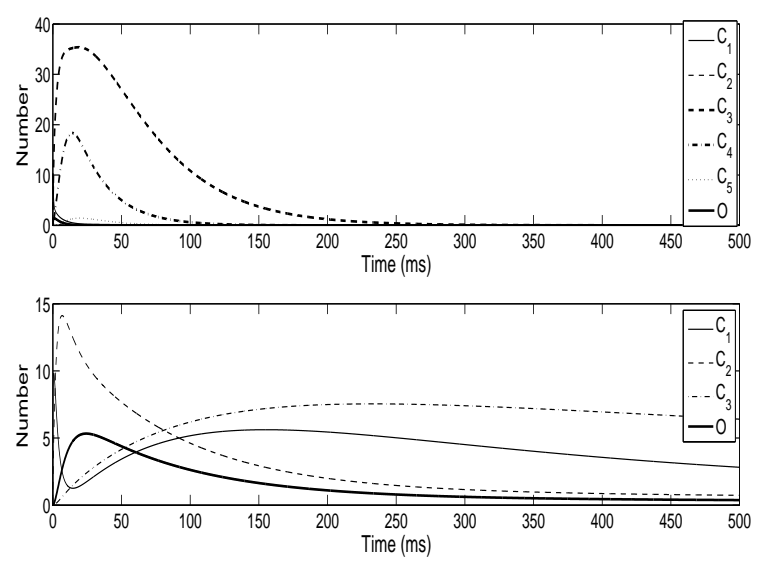

Fig. 6: Timecourse of (A) AMPAR and (B) NMDAR state occupancies.

However, we do not pursue this direction and settle with the density of 10000 GluTs per $\mu \mathrm{m}^{2}$, mainly to demonstrate that, for large quantal sizes (> 3000), even with such poor clearance times, the time-courses of the open states for both AMPARs and NMDARs are similar to those in a biological synapse.

\section{B. Evolution of Receptor States}

The evolution of the receptor states calculated by our algorithm, shown in Fig.6 and Fig.7, match quite well with the predictions of the Monte Carlo simulations of [25] except the artefacts introduced by the special setting of our AS. In the case of AMPAR, Fig.6(A) and Fig.7(A), the first state to attain its peak is $C_{1}$, which is followed by the peak of $C_{2}$. Next peaks the open state $O$, which occurs in less than $1 \mathrm{~ms}$, which is in agreement with [25]. In forthcoming times the receptors go into the desensitized states, and after $300 \mathrm{~ms}$ all receptors come back to the initial state $C_{0}$. The main difference compared to [25] is that, in our case the peaks of states $C_{1}, C_{2}$ and $O$ are very small. This is due to the fact that, in our case free glutamates start being captured by GluTs immediately
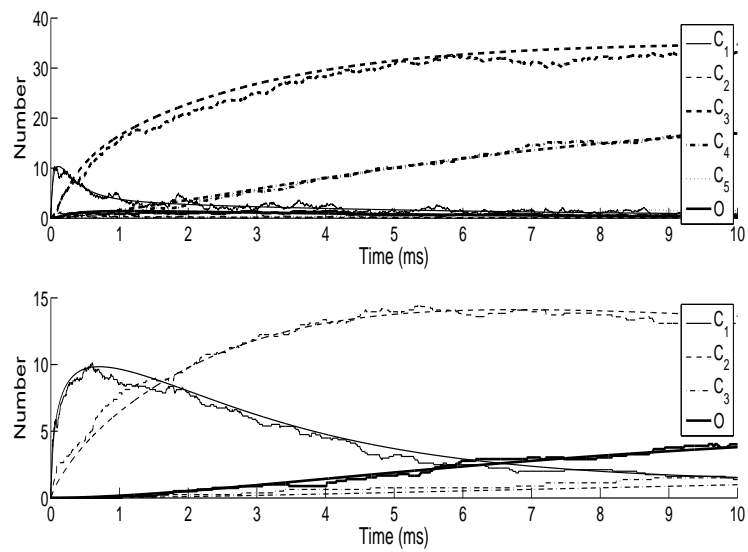

Fig. 7: Time-course of (A) AMPAR and (B) NMDAR state occupancies zoomed in. The results of our code and the Monte Carlo code (average of 100 experiments) are overlaid for comparison. 

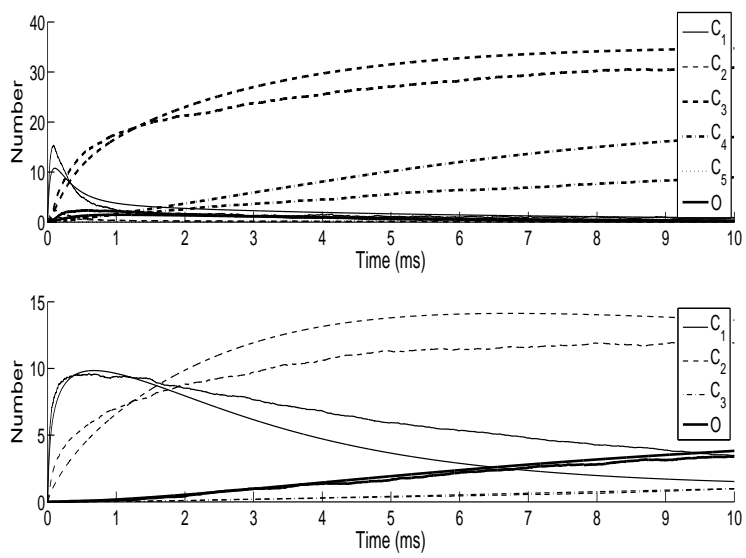

Fig. 8: Timecourse of (A) AMPAR and (B) NMDAR state occupancies zoomed in. The results of our code (thin lines) and MCell (thick lines, average of 100 experiments) are overlaid for comparison.

after the release, which decreases the glutamate concentrations near the receptors. Another artifact we have is a slower decay of desensitized AMPAR states, which is mainly due to the slow clearance of glutamates from AS mentioned before. For the case of NMDARs, Fig.6(B) and Fig.7(B), the agreement with [25] is stronger. The states follow exactly the same patterns, and the peak value and peak time of open NMDARs match quite well with a peak of 6 open NMDARs and a peak time of just above $20 \mathrm{~ms}$. However, again we observe a slower decay of desensitized NMDAR states due to the slow clearance of glutamates. In addition, our calculations verify the high glutamate affinity of NMDARs compared to AMPARs, [36], which is observed in the slower decay of NMDAR open states, and is due to the slow dissociation rate of glutamates from NMDARs.

\section{Variations in Quantal Size and Receptor Number}

In biological synapses quantal size and receptor number are suggested to be main contributors for synaptic plasticity, where, in particular, AMPAR number at individual synapses is thought to be the underlying factor in long-term synaptic plasticity, [37], [38], [39]. Our calculations support both of these hypotheses, and show that they are also valid for our AS. As seen in Fig.9, the peak value of open AMPARs increase with quantal size, whereas higher quantal size $(>3000)$ has no effect on the peak value and time of the NMDARs. This implies that, quantal size is a contributor to synaptic plasticity through AMPARs, and in a synapse containing only NMDARs quantal size has no effect on plasticity. On the other hand, receptor number contributes to synaptic plasticity through both AMPARs and NMDARs. In fact, Fig.10 shows that the ratio of the peak value of open AMPARs and NMDARs to the total number of AMPARs and NMDARs, respectively, is independent of the number of receptors in AS. This implies that the peak values of open AMPARs and NMDARs are both linear functions of the receptor number for any given quantal size. Another important difference between open AMPAR dynamics of the proposed AS and of the biological synapses
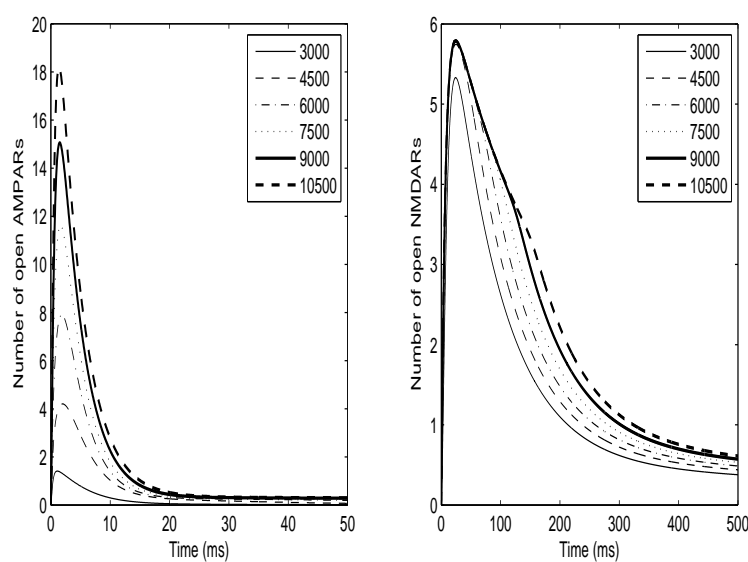

Fig. 9: Timecourse of open states of AMPARs (left) and NMDARs (right) for different values of quantal size.

can be observed from Fig.9. In [25], it is found that upon a vesicle release of quantal size 3000 , the number of open AMPARs decay to zero in around 200 ms. However, in Fig.9, we see that our calculations predict a decay to zero in less than $50 \mathrm{~ms}$. This difference seems to contradict to our finding that the clearance of glutamates from the AS with the assumed GluT density is slower than that of the biological synapses. However, it can be explained by the fast dissociation rate of glutamates from AMPARs, which in the biological setup causes glutamate molecules to associate with and dissociate from many AMPARs before they are able to leave the cleft, increasing the effective reserve of glutamate for AMPARs. However, in the proposed AS this phenomenon of multiple binding should be observed much less, as the glutamate molecules do not need to travel all the way across the cleft, but are sucked away from the AS through the presynaptic membrane, which is only $20 \mathrm{~nm}$ away. It is very interesting, however, that this phenomenon overcomes the difference in the glutamate clearance times. On the other hand, as the NMDARs have slow dissociation rate, such phenomenon does not apply
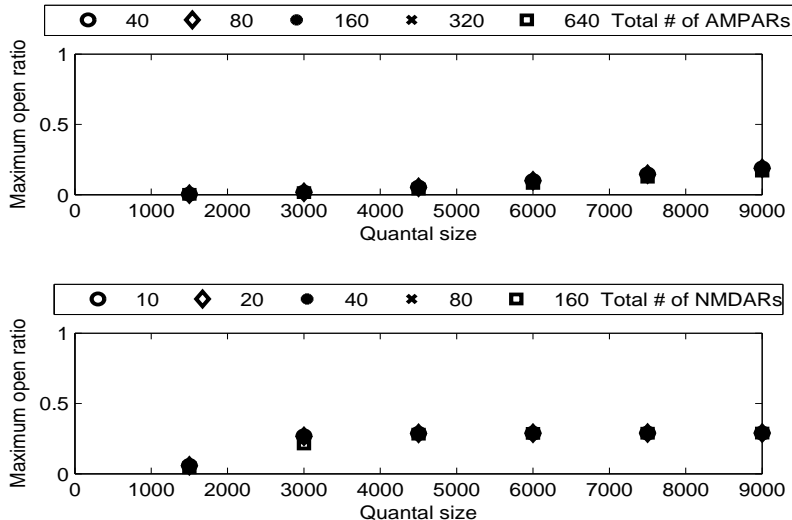

Fig. 10: Maximum open ratio for AMPARs (above) and NMDARs (below) as a function of quantal size plotted for different values of receptor numbers. 
to them, and in fact our calculations give worse decay rates for the number of open NMDARs compared to those of [25], which is, of course, due to the slow clearance from AS. In fact, in the second graph of Fig.9, in the decaying phase of open NMDARs, one can see a bump starting to appear for high quantal sizes, which is an artifact of slow clearance.

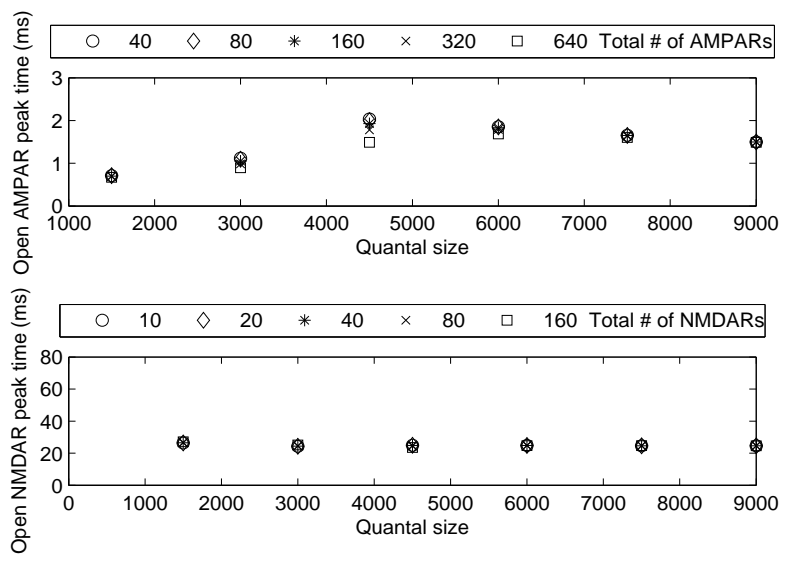

Fig. 11: Peak time for open AMPARs (above) and NMDARs (below) as a function of quantal size plotted for different values of receptor numbers.

Another interesting feature is the invariance of peak times for open AMPARs or NMDARs with respect to changes in receptor number, as in Fig.11. This phenomenon is due to the fact that the open states of both receptors peak in a short time compared to their dissociation rates, AMPARs with high dissociation rate peak very quickly $(0.5-1.5 \mathrm{~ms})$ and NMDARs peak rather slowly $(25-80 \mathrm{~ms})$ but have low dissociation rates, and consequently, up until the peak time glutamate bindings to receptors are almost independent from each other.

\section{Conclusions And Future Prospects}

In this paper, we have given a novel mathematical analysis of a proposed artificial synaptic channel. In the analysis, complex reaction dynamics of the communicating molecules are included. The analysis resulted in the development of a deterministic algorithm, which can calculate the expected behavior of various quantities of interest, such as the evolution of the states of various receptors, and the amount of free transmitters left in the cleft, etc. The success of this algorithm comes from the fact that, it is much faster than the widely used Monte Carlo algorithms.

Another novelty of this paper is the proposition of an AS. From the MC point of view, the proposed model of AS is theoretically shown to possess many desired properties of a biological synapse. As the most desired property, the timecourse of the open receptors are found to greatly match to that of a biological synapse, where the peak values and peak time of open receptors were a near exact match, except some discrepancy in the peak values of open AMPARs. The decay of open NMDARs was slower in the AS, and of AMPARs was faster, which, in view of the fact that as a design of nature AMPARs react fast and NMDARs slow, seems to be of little concern. Moreover, the AS showed great promise in terms of demonstrating synaptic plasticity, where the results of our algorithm suggests that the quantal size increases synaptic potency through its effect on AMPARs, and the synaptic potency linearly depends on the number of receptors for both AMPARs and NMDARs. The higher desensitization transients for both receptor types, observed due to longer glutamate clearance time of AS, is a source of synaptic depression, although this point was not elaborated upon.

In our investigation of the AS, we have pursued only the case of a single quantal release. However, an understanding of the dynamics of AS in the case of multiple quantal release is of paramount importance. Although it was not pursued here, it should be expected that, a multiple quantal release in AS upon an input spike train of high enough frequency (inside the range of frequencies observed in biological neural networks) should cause undesired temporal correlations between consecutive signals, mainly because of the high glutamate residence time of $\mathrm{AS}$ and the consequent slow decay of desensitized states of receptors. The analysis of the dynamics of the MC of our AS under multiple consecutive quantal inputs awaits further research.

The work done in this paper was solely interested in what happens inside the cleft of our AS, and that was done with molecules of biological origins, which are hard to synthesize or come by. One of the greatest challenges in realizing an AS remains to be devising its constituents. Other than the structural parts of the AS, there is a need to find appropriate candidate molecules for transmitters, transporters and receptors, which should be compatible with each other in terms of their reaction dynamics. Moreover, the transporters and receptors should be compatible with the structure, as to have the desired trans-membrane effects. There are ongoing studies to find candidate molecular machinery to achieve such tasks. For instance, in [40], the authors propose a promising molecular machinery capable of producing electrical output upon detection of molecules, which is a possible candidate for a receptor in AS design. Apart from the inside of the cleft of the AS, the transmitter pool and the injector as its main apparatus are hard design, even conceptually. Finally, the ultimate and most difficult challenge in realizing a true AS is in devising the MC between its parts in order to effectively regulate factors that contribute to synaptic plasticity.

\section{REFERENCES}

[1] D. Malak and O. B. Akan, "Molecular communication nanonetworks inside human body," Nano Communication Networks, vol. 3, no. 1, pp. 19-35, 2012.

[2] T. Nakano, M. J. Moore, F. Wei, A. V. Vasilakos, and J. Shuai, "Molecular communication and networking: Opportunities and challenges," IEEE transactions on nanobioscience, vol. 11, no. 2, pp. 135-148, 2012.

[3] T. Nakano, T. Suda, Y. Okaie, M. J. Moore, and A. V. Vasilakos, "Molecular communication among biological nanomachines: A layered architecture and research issues," IEEE transactions on nanobioscience, vol. 13, no. 3, pp. 169-197, 2014.

[4] P. Mathur, R. H. Nielsen, N. R. Prasad, and R. Prasad, "Conasense at nanoscale: Possibilities and challenges," Role of ICT for MultiDisciplinary Applications in 2030, vol. 47, p. 185, 2016.

[5] B. Picconi, G. Piccoli, and P. Calabresi, "Synaptic dysfunction in parkinsons disease," in Synaptic Plasticity. Springer, 2012, pp. 553572 . 
[6] O. B. Akan, H. Ramezani, T. Khan, N. A. Abbasi, and M. Kuscu, "Fundamentals of molecular information and communication science," in Proceedings of the IEEE. IEEE, 2016.

[7] D. Purves, G. J. Augustine, D. Fitzpatrick, L. C. Katz, A.-S. LaMantia, J. O. McNamara, and S. M. Williams, Neuroscience, 2nd Edition. Sinauer Associates, 2001

[8] H. Ramezani and O. B. Akan, "Synaptic channel model including effects of spike width variation," in Proceedings of the Second Annual International Conference on Nanoscale Computing and Communication. ACM, 2015, p. 11.

[9] D. Malak and O. B. Akan, "A communication theoretical analysis of synaptic multiple-access channel in hippocampal-cortical neurons," IEEE Transactions on communications, vol. 61, no. 6, pp. 2457-2467, 2013.

[10] E. Balevi and O. B. Akan, "A physical channel model for nanoscale neuro-spike communications," IEEE Transactions on Communications, vol. 61 , no. 3, pp. 1178-1187, 2013.

[11] T. Ohno, T. Hasegawa, T. Tsuruoka, K. Terabe, J. K. Gimzewski, and M. Aono, "Short-term plasticity and long-term potentiation mimicked in single inorganic synapses," Nature materials, vol. 10, no. 8, pp. 591595,2011

[12] L. Q. Zhu, C. J. Wan, L. Q. Guo, Y. Shi, and Q. Wan, "Artificial synapse network on inorganic proton conductor for neuromorphic systems," Nature communications, vol. 5, 2014.

[13] W. Xu, S.-Y. Min, H. Hwang, and T.-W. Lee, "Organic core-sheath nanowire artificial synapses with femtojoule energy consumption," Science advances, vol. 2, no. 6, p. e1501326, 2016.

[14] C. Zhang, Y.-T. Tai, J. Shang, G. Liu, K.-L. Wang, C. Hsu, X. Yi, $\mathrm{X}$. Yang, W. Xue, H. Tan et al., "Synaptic plasticity and learning behaviours in flexible artificial synapse based on polymer/viologen system," Journal of Materials Chemistry C, vol. 4, no. 15, pp. 32173223, 2016.

[15] M. C. Peterman, N. Z. Mehenti, K. V. Bilbao, C. J. Lee, T. Leng, J. Noolandi, S. F. Bent, M. S. Blumenkranz, and H. A. Fishman, "The artificial synapse chip: a flexible retinal interface based on directed retinal cell growth and neurotransmitter stimulation," Artificial organs, vol. 27, no. 11, pp. 975-985, 2003.

[16] J. C. Eccles, B. Katz, and S. W. Kuffler, "Effect of eserine on neuromuscular transmission," Journal of Neurophysiology, vol. 5, no. 3, pp. 211-230, 1942.

[17] J. C. Eccles and J. C. Jaeger, "The relationship between the mode of operation and the dimensions of the junctional regions at synapses and motor end-organs," Proceedings of the Royal Society of London B: Biological Sciences, vol. 148, no. 930, pp. 38-56, 1958.

[18] L. Wahl, C. Pouzat, and K. Stratford, "Monte carlo simulation of fast excitatory synaptic transmission at a hippocampal synapse," Journal of neurophysiology, vol. 75, no. 2, pp. 597-608, 1996.

[19] J. M. Bekkers and C. F. Stevens, "Nmda and non-nmda receptors are colocalized at individual excitatory synapses in cultured rat hippocampus," 1989.

[20] V. Kharazia and R. Weinberg, "Immunogold localization of ampa and nmda receptors in somatic sensory cortex of albino rat," The Journal of comparative neurology, vol. 412, no. 2, pp. 292-302, 1999.

[21] Y. Takumi, V. Ramírez-León, P. Laake, E. Rinvik, and O. P. Ottersen, "Different modes of expression of ampa and nmda receptors in hippocampal synapses," Nature neuroscience, vol. 2, no. 7, pp. 618-624, 1999.

[22] A. K. McAllister and C. F. Stevens, "Nonsaturation of ampa and nmda receptors at hippocampal synapses," Proceedings of the National Academy of Sciences, vol. 97, no. 11, pp. 6173-6178, 2000.

[23] C. Racca, F. A. Stephenson, P. Streit, J. D. B. Roberts, and P. Somogyi, "Nmda receptor content of synapses in stratum radiatum of the hippocampal cal area," The Journal of Neuroscience, vol. 20, no. 7, pp. 2512-2522, 2000

[24] N. Danbolt, F. Chaudhry, Y. Dehnes, K. Lehre, L. Levy, K. Ullensvang, and J. Storm-Mathisen, "Properties and localization of glutamate transporters," Progress in brain research, vol. 116, pp. 23-43, 1998.

[25] K. M. Franks, T. M. Bartol, and T. J. Sejnowski, "A monte carlo model reveals independent signaling at central glutamatergic synapses," Biophysical journal, vol. 83, no. 5, pp. 2333-2348, 2002.

[26] P. Jonas, G. Major, and B. Sakmann, "Quantal components of unitary epscs at the mossy fibre synapse on ca3 pyramidal cells of rat hippocampus." The Journal of Physiology, vol. 472, no. 1, pp. 615-663, 1993.

[27] R. Lester and C. E. Jahr, "Nmda channel behavior depends on agonist affinity," The Journal of neuroscience, vol. 12, no. 2, pp. 635-643, 1992.
[28] J. I. Wadiche, J. L. Arriza, S. G. Amara, and M. P. Kavanaugh, "Kinetics of a human glutamate transporter," Neuron, vol. 14, no. 5, pp. 10191027, 1995.

[29] J. S. Diamond and C. E. Jahr, "Transporters buffer synaptically released glutamate on a submillisecond time scale," The Journal of neuroscience, vol. 17, no. 12, pp. 4672-4687, 1997.

[30] J. R. Geiger, A. Roth, B. Taskin, and P. Jonas, "Glutamate-mediated synaptic excitation of cortical interneurons," in Ionotropic Glutamate Receptors in the CNS. Springer, 1999, pp. 363-398.

[31] J. R. Stiles, D. Van Helden, T. M. Bartol, E. E. Salpeter, and M. M. Salpeter, "Miniature endplate current rise times less than 100 microseconds from improved dual recordings can be modeled with passive acetylcholine diffusion from a synaptic vesicle," Proceedings of the National Academy of Sciences, vol. 93, no. 12, pp. 5747-5752, 1996.

[32] L. Longsworth, "Diffusion measurements, at 25, of aqueous solutions of amino acids, peptides and sugars," Journal of the American Chemical Society, vol. 75, no. 22, pp. 5705-5709, 1953.

[33] T. M. Bartol, A study of miniature endplate current generation at the vertebrate neuromuscular junction using electrophysiology and Monte Carlo simulation. Cornell University, May, 1992.

[34] M. B. Elowitz, M. G. Surette, P.-E. Wolf, J. B. Stock, and S. Leibler, "Protein mobility in the cytoplasm of escherichia coli," Journal of bacteriology, vol. 181, no. 1, pp. 197-203, 1999.

[35] R. J. Ellis, "Macromolecular crowding: obvious but underappreciated," Trends in biochemical sciences, vol. 26, no. 10, pp. 597-604, 2001.

[36] D. K. Patneau and M. L. Mayer, "Structure-activity relationships for amino acid transmitter candidates acting at n-methyl-d-aspartate and quisqualate receptors," The Journal of Neuroscience, vol. 10, no. 7, pp. 2385-2399, 1990.

[37] J. T. Isaac, R. A. Nicoll, and R. C. Malenka, "Evidence for silent synapses: implications for the expression of ltp," Neuron, vol. 15, no. 2, pp. 427-434, 1995.

[38] R. C. Carroll, D. V. Lissin, M. von Zastrow, R. A. Nicoll, and R. C. Malenka, "Rapid redistribution of glutamate receptors contributes to long-term depression in hippocampal cultures," Nature neuroscience, vol. 2, no. 5, pp. 454-460, 1999.

[39] Y. Hayashi, S.-H. Shi, J. A. Esteban, A. Piccini, J.-C. Poncer, and R. Malinow, "Driving ampa receptors into synapses by ltp and camkii: requirement for glur1 and pdz domain interaction," Science, vol. 287, no. 5461, pp. 2262-2267, 2000.

[40] M. Kuscu and O. Akan, "Modeling and analysis of sinw fet-based molecular communication receiver," IEEE Transactions on Communications, vol. 64 , no. 9 , pp. $3708-3721,2016$.

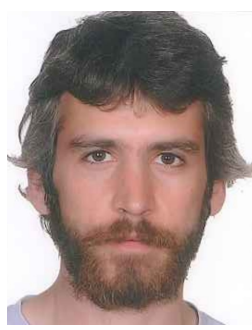

Bilgesu A. Bilgin [M'17] received his B.S. degree in Electrical and Electronics engineering from Middle East Technical University in 2008, M.Sc. and Ph.D. degrees in mathematics from Koc University, in 2011 and 2015, respectively. He has worked as a postdoctoral fellow in Next-generation and Wireless Communications Laboratory at Koc University from 2015 to 2017. Currently, he is working as a postdoctoral research associate in the Engineering Department at the University of Cambridge. His research interests include molecular communication, intrabody nanonetworks, partial differential equations and dynamical systems.

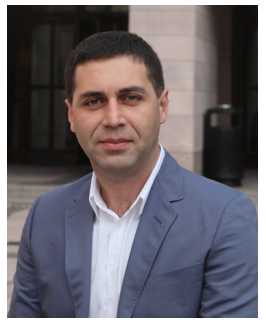

Ozgur B. Akan [M'00-SM'07-F'16] received PhD degree from the School of Electrical and Computer Engineering, Georgia Institute of Technology, USA, in 2004. He is currently with the Electrical Engineering Division, Department of Engineering, University of Cambridge, UK and the Department of Electrical and Electronics Engineering, Koc University, Istanbul, Turkey. His research interests include wireless, nano, molecular communications, and Internet of Everything. 